\title{
Increased Hippocampal Default Mode Synchronization during Rest in Middle-Aged and Elderly APOE $\varepsilon 4$ Carriers: Relationships with Memory Performance
}

\author{
Erling T. Westlye, ${ }^{1}$ Arvid Lundervold, ${ }^{1,2}$ Helge Rootwelt, ${ }^{3}$ Astri J. Lundervold, ${ }^{4,5}$ and Lars T. Westlye ${ }^{6}$ \\ ${ }^{1}$ Neuroinformatics and Image Analysis Laboratory, Department of Biomedicine, University of Bergen, 5009 Bergen, Norway, ${ }^{2}$ Department of Radiology, \\ Haukeland University Hospital, 5021 Bergen, Norway, ${ }^{3}$ Department of Medical Biochemistry, Oslo University Hospital, Rikshospitalet, 0424 Oslo, Norway, \\ ${ }^{4}$ Department of Biological and Medical Psychology, University of Bergen, 5009 Bergen, Norway, ${ }^{5}$ Kavli Research Centre for Aging and Dementia, \\ Haraldsplass Deaconess Hospital, 5009 Bergen, Norway, and ${ }^{6}$ Center for the Study of Human Cognition, Department of Psychology, University of Oslo, 0317 \\ Oslo, Norway
}

The apolipoprotein (APOE) $\varepsilon 4$ allele is a strong genetic risk factor for Alzheimer's disease (AD). Intrinsic fluctuations of brain activity measured by fMRI during rest may be sensitive to AD-related neuropathology. In particular, functional connectivity of the default-mode network (DMN) has gained recent attention as a possible biomarker of disease processes and associated memory decline in AD. Here, we tested the hypothesis of APOE-related alterations in DMN functional connectivity in 95 healthy individuals between 50 and 80 years of age, including 33 carriers of the $\varepsilon 4$ allele. Based on previous studies, we hypothesized increased hippocampal DMN synchronization in APOE $\varepsilon 4$ carriers. This was supported using independent component analysis in combination with a dual-regression approach for analysis of resting state data. Whole-brain analysis suggested effects also in other areas, including the posterior cingulate cortex, parietal cortex, and parahippocampal regions. DMN synchronization showed a negative correlation with performance on a test of memory functioning, suggesting a neurocognitive significance of the brain activity patterns during rest. Our findings indicate that increased genetic vulnerability for AD is reflected in increased hippocampal DMN synchronization during rest several years before clinical manifestation. We propose that the results reflect $\varepsilon 4$-related failure in hippocampal decoupling, which might elevate the total hippocampal metabolic burden and increase the risk of cognitive decline and $\mathrm{AD}$. The results provide an important confirmation of specific genotype effects on intrinsic fluctuations and support the use of functional connectivity indices as imaging-derived endophenotypes in the emerging field of imaging genetics.

\section{Introduction}

Resting state functional connectivity (RSFC) studies have revealed specific functional neuronal networks (Biswal et al., 1995), including low-level sensorimotor and higher-order attention and cognitive control networks (Zhang and Raichle, 2010). Resting state networks (RSNs) are identifiable in the absence of contextual demands, but show high correspondence with task-evoked networks (Smith et al., 2009). The coherence and spatial distribution of the RSNs persist across wakefulness and sleep and also

\footnotetext{
Received March 10, 2011; accepted April 7, 2011

Author contributions: E.T.W., A.L., A.J.L., and L.T.W. designed research; E.T.W. and A.J.L. performed research; E.T.W., H.R., A.J.L., and L.T.W. analyzed data; E.T.W. and L.T.W. wrote the paper.

The authors declare no competing financial interests.

This study was supported by Grant 911397 from the Western Norway Regional Health Authority to A.J.L., by Grant 911593 from Helse Vest (MedViz/Quantitative Brain MR Imaging in Aging and Neurodegenerative Disorders) to A.L., and by the Research Council of Norway to L.T.W. We thank Dr. Jonn-Terje Geitung and the Department of Radiology at the Haraldsplass Deaconess Hospital for providing access to the MRI facility and the participants who made this study possible.

Correspondence should be addressed to Dr. Erling Tjelta Westlye, Neuroinformatics and Image Analysis Laboratory, Department of Biomedicine, University of Bergen, Jonas Lies vei 91, N-5009 Bergen, Norway. E-mail: Erling.Westlye@student.uib.no.

DOI:10.1523/JNEUROSCI.1230-11.2011

Copyright $\odot 2011$ the authors $\quad 0270-6474 / 11 / 317775-09 \$ 15.00 / 0$
}

under anesthesia (Zhang and Raichle, 2010). Demonstrations of intrinsic functional connectivity in the monkey (Vincent et al., 2007; Margulies et al., 2009) and infant brain (Fransson et al., 2007,2011 ) support that the patterns of RSFC are evolutionarily conserved traits of brain functional architecture.

A heavily investigated RSN is the default-mode network (DMN) (Raichle et al., 2001). Altered DMN RSFC has been observed in clinical conditions including mild cognitive impairment and Alzheimer's disease (AD) (Greicius et al., 2004; Sorg et al., 2007). The close neuroanatomical relations between the DMN and the loci of AD neuropathology are compelling. Based on the notion that early $\mathrm{AD}$ pathology forms preferentially throughout areas that make up the DMN (Braak and Braak, 1991; Buckner et al., 2005), it has been suggested that DMN metabolism may be related to disease processes and associated memory decline (Buckner et al., 2008). In line with this hypothesis, several studies have reported disease- and age-related variability in the intrinsic fluctuations of the DMN (Greicius et al., 2004; Damoiseaux et al., 2008).

Carriers of the APOE $\varepsilon 4$ allele have a twofold to threefold increased risk of developing late-onset $\mathrm{AD}$ if they are heterozygous and $\sim 12$-fold if homozygous for the $\varepsilon 4$ allele (Roses, 1996; 
Bertram et al., 2007). Evidence linking APOE $\varepsilon 4$ to RSFC in healthy individuals is limited, and previous studies either did not include a pure resting condition (Persson et al., 2008; Fleisher et al., 2009a; Pihlajamäki and Sperling, 2009) or included relatively few participants (Filippini et al., 2009; Fleisher et al., 2009a), limiting the statistical power. Filippini et al. (2009) demonstrated altered RSFC in retrosplenial, medial-prefrontal, and medial temporal lobe (MTL) regions including the hippocampi in young (20-35 years old) subjects carrying the $\varepsilon 4$ allele. Interestingly, $\varepsilon 4$ carriers also showed increased hippocampal activation during memory encoding, but neither result was explained by differences in memory performance, brain morphology, or resting cerebral blood flow (Filippini et al., 2009). Effects of APOE $\varepsilon 4$ on RSFC have not been demonstrated in middle-aged and elderly individuals using independent component analysis and dual regression.

Therefore, the aim of the present study was to test the hypothesis of specific $\varepsilon 4$-related alterations in DMN synchronization in a sample of 95 healthy individuals between 50 and 80 years of age, including 34 carriers of the $\varepsilon 4$ allele. Based on one study using a similar analytical approach (Filippini et al., 2009), we hypothesized that MTL regions would show increased DMN synchronization in carriers compared with noncarriers beyond what can be explained by hippocampal volumes and cortical gray matter (GM) density. We further hypothesized that MTL-DMN synchronization during rest would be inversely associated with performance on a standardized test of memory function.

\section{Materials and Methods}

Subjects. Healthy individuals were invited through an advertisement to take part in the first wave of a longitudinal study of cognitive aging. Subjects with a history of substance abuse, present neurologic or psychiatric disorder, or other significant medical conditions were excluded from the study. In this first wave, all participants were examined according to an extensive neuropsychological test protocol, including assessment of memory function, and delivered blood for genotyping (see below). The present study included participants who were invited to a follow-up study after 3 or 4 years, of which 112 participants took part in an MRI examination including 3D MRI and resting state fMRI. All subjects' scans were evaluated by an experienced neuroradiologist. Brain tumors, cysts, recent infarctions, or gross regional or global signal abnormalities were exclusion criteria. No participants were excluded based on the neuroradiological evaluation. Twelve subjects were excluded because of lack of genotyping data. In line with previous studies (Persson et al., 2008; Filippini et al., 2009), two individuals with genotype $\varepsilon(2,4)$ were excluded as the $\varepsilon 2$ variant is reported to protect against cognitive decline (Wilson et al., 2002) and AD (Benjamin et al., 1994). Further, two individuals with MMSE $<27$ were excluded (Folstein et al., 1975). One final participant was excluded because of low estimated general intellectual abilities ( $\mathrm{IQ}=64$ ). The final sample thus comprised 95 healthy participants aged $50-80$ years $[$ mean age $=63.8$ years $(\mathrm{SD}=$ 7.2), 61 women], including 33 carriers of the APOE $\varepsilon 4$ allele. Group characteristics are shown in Table 1 . There were no significant differences between the genotype groups with respect to sex (Fischer's exact twosided test, $p=0.66$ ) or age (independent two-sample $t$ test, $p=0.347$ ).

All subjects provided informed consent according to the Declaration of Helsinki. The project was approved by the Regional Committee for Research Ethics of Western Norway, and a biobank for storage of personalized data was approved by the Department of Health.

Measures of memory function. All subjects were tested with the Norwegian translation of the California Verbal Learning Test, second version (CVLT-II) (Delis et al., 1987). A list of 16 words (List A) was presented five times. Immediately after the fifth trial, the participants were read a new list (List B) and asked to recall it. Then, the participants were asked to recall the words from List A, immediately after the recall of List B and $\sim 20$ min later (the long-delayed condition). Subsequently, a recognition
Table 1. Group characteristics

\begin{tabular}{|c|c|c|c|c|}
\hline Variable & $\mathrm{APOE} \in 4-$ & $\mathrm{APOE} \in 4+$ & $t$ & $p$ \\
\hline \multicolumn{5}{|l|}{ Demographic } \\
\hline$n$ & 62 & 33 & & \\
\hline Age & $64.4(7.0)$ & $62.6(7.6)$ & 1.180 & 0.241 \\
\hline Sex & $21 \mathrm{M} / 41 \mathrm{~F}$ & $13 \mathrm{M} / 20 \mathrm{~F}$ & & 0.656 \\
\hline MMSE & $28.8(0.91)$ & $29.1(0.97)$ & -1.108 & 0.271 \\
\hline \multicolumn{5}{|l|}{ Volumetrics } \\
\hline L hippocampus (mm ${ }^{3}$ ) & $3300.4(400.8)$ & $3370.4(229.2)$ & -0.396 & 0.693 \\
\hline R hippocampus $\left(\mathrm{mm}^{3}\right)$ & $3275.4(398.3)$ & $3401.5(331.7)$ & -1.076 & 0.285 \\
\hline Mean hippocampus (mm ${ }^{3}$ ) & $3287.9(387.0)$ & $3386.0(262.3)$ & -0.787 & 0.433 \\
\hline $\mathrm{ICV}\left(\mathrm{mm}^{3}\right)$ & $\begin{array}{l}1.333 \times 10^{6} \\
\left(0.143 \times 10^{6}\right)\end{array}$ & $\begin{array}{l}1.370 \times 10^{6} \\
\left(0.128 \times 10^{6}\right)\end{array}$ & -1.233 & 0.221 \\
\hline \multicolumn{5}{|l|}{ CVLT raw scores } \\
\hline Learning & $56.0(10.8)$ & $56.1(11.9)$ & -0.018 & 0.985 \\
\hline Learning—age adjusted & & & 0.357 & 0.734 \\
\hline Recall & $12.8(2.87)$ & $12.6(2.78)$ & 0.060 & 0.952 \\
\hline Recall—age adjusted & & & 0.583 & 0.561 \\
\hline Recognition discriminability & $3.45(0.64)$ & $3.17(0.83)$ & 1.833 & 0.070 \\
\hline $\begin{array}{l}\text { Recognition discriminability- } \\
\text { age adjusted }\end{array}$ & & & 2.269 & 0.026 \\
\hline
\end{tabular}

Mean of MMSE and other variables are given with SD in parentheses. The volumes for left $(\mathrm{L})$ hippocampus, right $(\mathrm{R})$ hippocampus, and mean hippocampi are raw volumes not adjusted for intracranial volume (ICV). The respective and $p$ values are from the $t$ test on the standardized residuals when using ICV as covariate in a regression fit. For sex we used Fisher's exact test. Mean (SD) is reported separately for the three measures of verbal memory function derived from the CVLT. The respective $t$ and $p$ values are from two-tailed independent-samples $t$ tests. The ageadjusted $t$ and $p$ values are from the $t$ test on the standardized residuals from a regression fit with age as covariate. F, Female; M, male.

\section{Table 2. APOE genotype distribution}

\begin{tabular}{lrl}
\hline APOE allele & $n$ & Sex \\
\hline 2,3 & 6 & $3 \mathrm{M} / 3 \mathrm{~F}$ \\
$2,4^{a}$ & 2 & $1 \mathrm{M} / 1 \mathrm{~F}$ \\
3,3 & 56 & $18 \mathrm{M} / 38 \mathrm{~F}$ \\
3,4 & 26 & $11 \mathrm{M} / 15 \mathrm{~F}$ \\
4,4 & 7 & $2 \mathrm{M} / 5 \mathrm{~F}$ \\
\hline
\end{tabular}

${ }^{a}$ The two subjects with this allele configuration were excluded from the DMN analysis (see Materials and Methods for rationale). F, Female; $M$, male.

trial was presented in which the participants were asked to identify the 16 items from List A from a larger list that contained various distractor items. In the present study, we included the following three CVLT measures: learning, defined as the number of hits across the five learning trials; recall, defined as the number of words recalled in the free longdelayed condition; and recognition discriminability, reflecting the difference in SD units between the examinee's hit rate and false-positive rate $\left(d^{\prime}\right)$ (Delis et al., 1987).

Genotyping. The whole-blood samples were collected at the Department of Biological and Medical Psychology at the University of Bergen by a bioengineer right after the neuropsychological examination; samples were immediately frozen and sent to Department of Medical Biochemistry, Oslo University Hospital, Rikshospitalet, Norway, for ApoE analysis. Genotyping was performed by real-time PCR with allele-specific fluorescence energy transfer probes and melting curve analysis on the LightCycler system (Roche Diagnostics). DNA was extracted from $300 \mu \mathrm{l}$ of whole blood using MagNA Pure LC DNA Isolation Kit-Large Volume on the MagNA Pure LC (Roche), eluted and diluted to $1 \mathrm{ml}$, of which $5 \mu \mathrm{l}$ was applied in each assay. Typing of the APOE $\varepsilon 2, \varepsilon 3$, and $\varepsilon 4$ genotypes was performed using the LightCycler APOE Mutation Detection Kit (Roche). The assay was performed as specified by the supplier, except for scaling down the total assay volume from 20 to $10 \mu$ l. The laboratory participates in an external quality assurance program (Equalis) that includes APOE genotyping. Allele frequency combinations are shown in Table 2. The genotype frequencies are in Hardy-Weinberg equilibrium, and the observed carrier frequency of $34.7 \%$ homozygotes or heterozygotes for $\varepsilon 4$ is in accordance with the known high frequency of this allele in the northern European population (Gerdes, 2003). 
MRI acquisition. Imaging was performed on a 1.5 T GE Signa Echospeed Scanner, using a standard eight-channel head coil. For volumetry, we obtained two T1-weighted 3D inversion recovery-prepared fast spoiled gradient-recalled acquisitions in a steady state in succession (to improve signal-to-noise ratio and segmentation accuracy), with the following pulse sequence parameters: repetition time (TR)/echo time (TE)/ inversion time $(\mathrm{TI}) /$ flip angle $(\mathrm{FA})=9.11 \mathrm{~ms} / 1.77 \mathrm{~ms} / 450 \mathrm{~ms} / 7^{\circ}$; voxel size, $0.94 \times 0.94 \times 1.40 \mathrm{~mm} ; 124$ (sagittal) slices; scan time $\sim 6 \mathrm{~min}$. Resting state BOLD fMRI data were collected for each subject with a T2*-weighted single-shot gradient echo EPI sequence with the following parameters: TR/TE/FA $=2000 \mathrm{~ms} / 50 \mathrm{~ms} / 90^{\circ}$; voxel size, $3.75 \times 3.75 \times$ $5.0 \mathrm{~mm} ; 256$ volumes ( 25 axial slices); scan time $\sim 8 \mathrm{~min}$. Participants were instructed to lie still in the scanner with their eyes closed, to think of nothing in particular, and not to fall asleep. Cushions and headphones were used to reduce subject motion and scanner noise.

MRI processing and analysis. The T1-weighted 3D MR images were processed using FreeSurfer (http://surfer.nmr.mgh.harvard.edu) enabling automated volumetric segmentation of various neuroanatomical structures including the hippocampi. The segmentation procedure has been described previously in detail (Dale et al., 1999; Fischl and Dale, 2000; Fischl et al., 2001, 2002, 2004). Briefly, the processing scheme includes motion correction and averaging of the two acquisitions, intensity normalization (Sled et al., 1998), removal of nonbrain tissue using a hybrid watershed/surface deformation procedure (Ségonne et al., 2004), automated Talairach transformation, segmentation of the subcortical white matter and deep gray matter volumetric structures (including hippocampus, amygdala, caudate, putamen, and ventricles) (Fischl et al., 2002, 2004), tessellation of the gray/white matter boundary, automated topology correction (Fischl et al., 2001; Ségonne et al., 2007), and surface deformation following intensity gradients to optimally place the gray/ white and gray/CSF borders at the location where the greatest shift in intensity defines the transition to the other tissue class (Dale and Sereno, 1993; Dale et al., 1999; Fischl and Dale, 2000).

Individual GM density maps were computed from the T1-weighted 3D MR images using the script feat_gm_prepare, which is distributed with FSL, and FMRIB's Automated Segmentation Tool (FAST) (Zhang et al., 2001). Briefly, individual maps were registered into standard space, smoothed to suit the functional acquisition $(\sigma=2.63)$, demeaned, and added as voxelwise explanatory variables in the statistical models (Filippini et al., 2009).

Resting-state fMRI analysis of the 256 volumes in time were performed using Multivariate Exploratory Linear Optimized Decomposition into Independent Components (MELODIC) (Beckmann et al., 2005) implemented in FSL (Smith et al., 2004; Woolrich et al., 2009) (http://www. fmrib.ox.ac.uk/fsl). Individual processing included discarding the first four volumes to let the scanner reach equilibrium due to progressive saturation, motion correction, spatial smoothing using a Gaussian kernel of FWHM of $6 \mathrm{~mm}$, and high-pass temporal filtering equivalent to $150 \mathrm{~s}$ $(0.007 \mathrm{~Hz})$. fMRI volumes were registered to the subject's skull-stripped T1-weighted scan processed in FreeSurfer using FMRIB's Linear Image Registration Tool (FLIRT) (Jenkinson and Smith, 2001; Jenkinson et al., 2002). The T1-weighted volume was registered and warped to Montreal Neurological Institute 152 standard space (MNI-152) using FMRIB's Nonlinear Image Registration Tool (FNIRT) (Andersson et al., 2007a, 2007b), and the resulting nonlinear transform was applied to the fMRI data. Next, the processed functional data were temporally concatenated across subjects to create a single $4 \mathrm{D}$ dataset.

The between-subjects analysis was performed using dual regression (Filippini et al., 2009), allowing for voxelwise comparisons of resting functional connectivity. This specific method has been proven more consistent and reliable than template-matching approaches in its ability to estimate individual-level RSNs from group-level independent component analysis (gICA) spatial maps (Zuo et al., 2010). The procedure comprises three steps: First, gICA is applied to the concatenated restingstate data. RSFC is a relatively new area of research, and the optimal number of components reflecting the true biological dimensionality in the brain is currently unknown. Thus, the choice of dimensionality in resting fMRI studies is arbitrary and will to a certain degree influence the spatial distribution of the DMN. Here, underfitting and overfitting of the components were minimized by using the Laplace approximation to the Bayesian evidence for a probabilistic principal component (Minka, 2000; Beckmann and Smith, 2004).

Second, the dual-regression algorithm (Filippini et al., 2009) is applied to identify subject-specific time courses and spatial maps. The procedure employs a set of gICA spatial maps in a linear model fit against the individual fMRI dataset. This results in matrices describing the temporal dynamics of the corresponding RSN for each of the 95 subjects. These time course matrices are normalized by their variance and used in a linear model fit against the individual fMRI dataset. This temporal regression results in subject-specific spatial maps. These maps reflect degree of synchronization, which is not simply a coherence measure, as they reflect both amplitude and coherence across space (Roosendaal et al., 2010). Here, 19 gICA maps reflecting various RSNs were included in the dual regression.

Third, the different synchronization maps are collected across subjects into 4D files (one file per original ICA map, with the fourth dimension being subject identification) and submitted to voxel-based statistical testing (see below). To explore potential bias inflicted by the unequal group sizes, we correlated the component in question with the analogous IC estimated using equal-sized groups ( $14 \mathrm{men} / 20$ women in each, no group differences with respect to age and sex). The two components showed very high correlation (Pearson's $r=0.90$ ). Thus, we performed the dual regressions on the gICA maps estimated from the full sample ( 95 subjects). Effects of APOE status were tested within the DMN, and we performed similar analysis on a control network (primary visual network), where we did not expect to see any effects of genotype, to test for specificity.

Statistical analysis. Effects of APOE genotype on functional synchronization were tested voxelwise by means of nonparametric permutation testing using 5000 permutations (Nichols and Holmes, 2002) as implemented in randomise, part of FSL (Smith et al., 2004). Threshold-free cluster enhancement was used to avoid the arbitrariness in defining smoothing level and initial cluster-forming thresholds (Smith and Nichols, 2009). Effects were regarded significant at $p<0.05$, corrected for multiple comparisons across space. We statistically accounted for effects of age, sex, and hippocampal volumes by including these variables as subjectwise covariates in the statistical models. Here, we used standardized residuals of mean volume of left and right hippocampus after regressing out estimated intracranial volume (Buckner et al., 2004). To account for effects of other structural differences between the two groups, we used GM density maps as voxelwise covariates. The three independent components spanning the DMN and the component spanning visual cortex were dealt with separately for the third part of dual regression.

Because of our a priori hypothesis of specific MTL effects of APOE status and to decrease the number of tests, we restricted our initial analyses to MTL regions (hippocampus + amygdala) as defined by the digitalized probabilistic Harvard-Oxford subcortical atlas provided with FSL. The masks were thresholded at 5\% probability level, combined, and used as spatial masks in the statistical analysis. Next, to explore APOErelated variability outside the restricted masks, we performed full-brain explorative follow-up analysis. We computed Cohen's $d$ (Cohen, 1992) in each of the significant clusters to characterize the regional variability in effect sizes.

Statistical analyses of the three verbal memory measures were performed using the package PASW Statistics 18.0. To explore the neurocognitive significance of the RSFC, DMN synchronization within the clusters showing significant effect of group ( $p<0.05$, corrected) was correlated (Pearson's $r$ ) with memory performance while partialing out age and sex. For sociodemographic variables, neuropsychological test scores, and brain structure volumes, we used independent-samples $t$ tests. Pearson's $\chi^{2}$ tests were used for categorical variables (sex and APOE status).

\section{Results}

\section{Verbal memory function}

Mean CVLT-II scores on the learning, recall, and recognition discriminability measures within each genotype group are presented in Table 1. Independent-samples $t$ tests revealed no statis- 
tically significant differences between the two APOE groups on learning and recall. However, we found decreased recognition discriminability scores in carriers compared with noncarriers $(t=$ $2.27, p<0.03$ ), indicating superior performance in noncarriers. We also found a significant negative effect of age on discriminability scores across the APOE groups $(r=-0.29, p<0.01)$. Follow-up analysis showed similar effects of age within noncarriers $(r=-0.38$, $p<0.005)$ and carriers $(r=-0.24, p>0.05)$ of the $\varepsilon 4$ allele. Fisher's $z$ test revealed no significant difference in age effects on performance between the APOE groups $(z=-0.71, p>0.05)$.

\section{Hippocampal volumes}

Table 1 summarizes mean volume of the left and right hippocampus within and across groups and genders. Mean volume of the left and right hippocampus for noncarriers was 3300.4 (SD = $400.8) \mathrm{mm}^{3}$ and $3275.4(\mathrm{SD}=398.3) \mathrm{mm}^{3}$, respectively. Mean volume for the left and right hippocampus for carriers was 3370.4 $(\mathrm{SD}=229.2) \mathrm{mm}^{3}$ and $3401.5(\mathrm{SD}=331.7) \mathrm{mm}^{3}$, respectively. Independent-samples $t$ tests revealed no significant effect of APOE $\varepsilon 4$ on hippocampus volumes ( $p$ values $>0.05$ ).

\section{Independent component analysis of $\mathrm{AMRI}$ data}

Spatial gICA (MELODIC) produced 41 independent components estimated using the Laplace approximation to the Bayesian evidence for a probabilistic principal component (Beckmann and Smith, 2004). By visual inspection, 22 of the components were identified as artifactual, caused by variation in subjects' head sizes, head movement, or other physiological fluctuations. By visual inspection, we identified the DMN as the spatial map comprising PFC, ACC, and posterior cingulate cortex (PCC), lateral parietal cortex (LPC), inferior and medial temporal gyri, and thalamic nuclei extending to MTL regions (Raichle et al., 2001; Greicius et al., 2003; Fox et al., 2005; Damoiseaux et al., 2006; Boly et al., 2008). Figure $1 A$ shows the three independent components identified as DMN constituents, and Figure $1 B$ shows the primary visual component.

\section{DMN connectivity}

Figure $1 C$ shows the spatial distribution of the $p$ statistics from the main MTL analysis in the posterior DMN comprising the precuneus, ACC, PCC, and the thalamus. Significantly increased synchronization in carriers compared with noncarriers was found in large parts of the right MTL ( $p<0.05$, corrected). Clusterwise mean values for carriers and noncarriers and effect sizes (Cohen's $d$ ) are shown in Table 3. Briefly, we found moderate regional variability in effect sizes. The largest effect size was found in the right hippocampus/amygdala $(d=1.23)$ and the smallest in the left amygdala $(d=0.81)$. No significant effects of APOE $\varepsilon 4$ status were shown in the control RSN (primary visual network).

Figure $1 D$ shows the results from the explorative full-brain analysis. Increased synchronization in carriers was found in the same DMN component in several areas, mostly in the right hemisphere, including amygdala, hippocampus, PCC, and precuneus. See Table 3 for details regarding the spatial distribution and clusterwise effect sizes. The largest effect size was found in the insular and temporal pole $(d=1.23)$ and the smallest in occipital fusiform and lingual gyrus $(d=0.61)$. None of the other DMN components showed effects of the APOE $\varepsilon 4$ genotype. Including GM density maps as voxelwise covariates in the statistical models only moderately altered the results. Full-brain analysis using age, sex, hippocampal volumes, and GM maps as covariates reduced the total number of significant voxels from 622 to 399, and the number of clusters increased from 9 to 10. The spatial distribution was very similar to what is displayed in Figure $1 D$, indicating that the effects of APOE status on RSFC in the posterior DMN cannot be explained by group differences in GM density.

\section{Correlation between DMN synchronization and memory performance}

We found a significant negative correlation $(r=-0.32, p<0.002)$ between recognition discriminability and RSFC in the hippocampal cluster from the main analysis (Fig. 1C), while partialing out age and sex. Figure 2 displays a scatter plot of discriminability performance as a function of hippocampal RSFC in the total sample, indicating decreasing DMN synchronization with increasing recognition discriminability.

To examine other regions outside hippocampus, we correlated synchronization within all clusters from the full-brain analysis with the recognition discriminability performance. Here, we also found a significant negative relationship $(r=-0.27, p<$ 0.01 ). To further explore the spatial variability, we correlated all significant clusters from the full-brain analysis with the recognition discriminability independently. All clusters showed a weak negative relationship between synchronization and the performance on memory, but only the correlations in right precentral gyrus $(r=-0.21, p<0.05)$ and right frontal orbital cortex $(r=$ $-0.20, p<0.05)$ were significant.

\section{Effect of age on RSFC within the APOE $\varepsilon 4$ carrier group}

We tested whether the RSFC in hippocampus and amygdala would fall off with age or remain high within the APOE $\varepsilon 4$ carrier group. Briefly, we found no effects of increasing age in this group. Voxelwise statistics were performed using only the independent component spanning the posterior DMN and were regarded as significant at $p<0.05$, corrected for multiple comparisons. Results revealed no significant effects of age across APOE carrier groups. No significant effect was found when contrasting with APOE3 $\times$ age-negative and positive slopes-nor APOE $4 \times$ age - negative and positive slopes. We found one significant voxel when contrasting APOE $3 \times$ age $>$ APOE $4 \times$ age, but correcting for outliers removed these effects. General linear model analysis on each reported cluster (Table 3) revealed no significant interaction of age and APOE $\varepsilon 4$ carrier status after outlier corrections.

\section{Discussion}

We tested the effects of APOE $\varepsilon 4$ on DMN synchronization in 95 healthy individuals between 50 and 80 years of age using gICA and dual regression. In line with our hypothesis, we demonstrated increased hippocampal synchronization in carriers relative to noncarriers of the $\varepsilon 4$ allele in an RSN spanning the posterior DMN. Whole-brain analysis revealed extended effects into the PCC, parietal, and parahippocampal regions. The lack of effects in other RSNs indicates specific effects of APOE on the functional coherence of the posterior DMN, including MTL and retrosplenial cortices, known to be early targets in AD. Furthermore, we report a negative relationship between memory performance and resting hippocampal DMN synchronization, which indicates that intrinsic functional connectivity patterns have neurocognitive correlates. Implications of the results are discussed in detail below.

APOE $\varepsilon 4$ is linked to accelerated brain aging and disease (Small et al., 2000; Deary et al., 2002; Wilson et al., 2002; Bertram et al., 2007; Espeseth et al., 2008). As a risk factor for AD, there is evidence suggesting that the role of the apolipoprotein in amyloid 

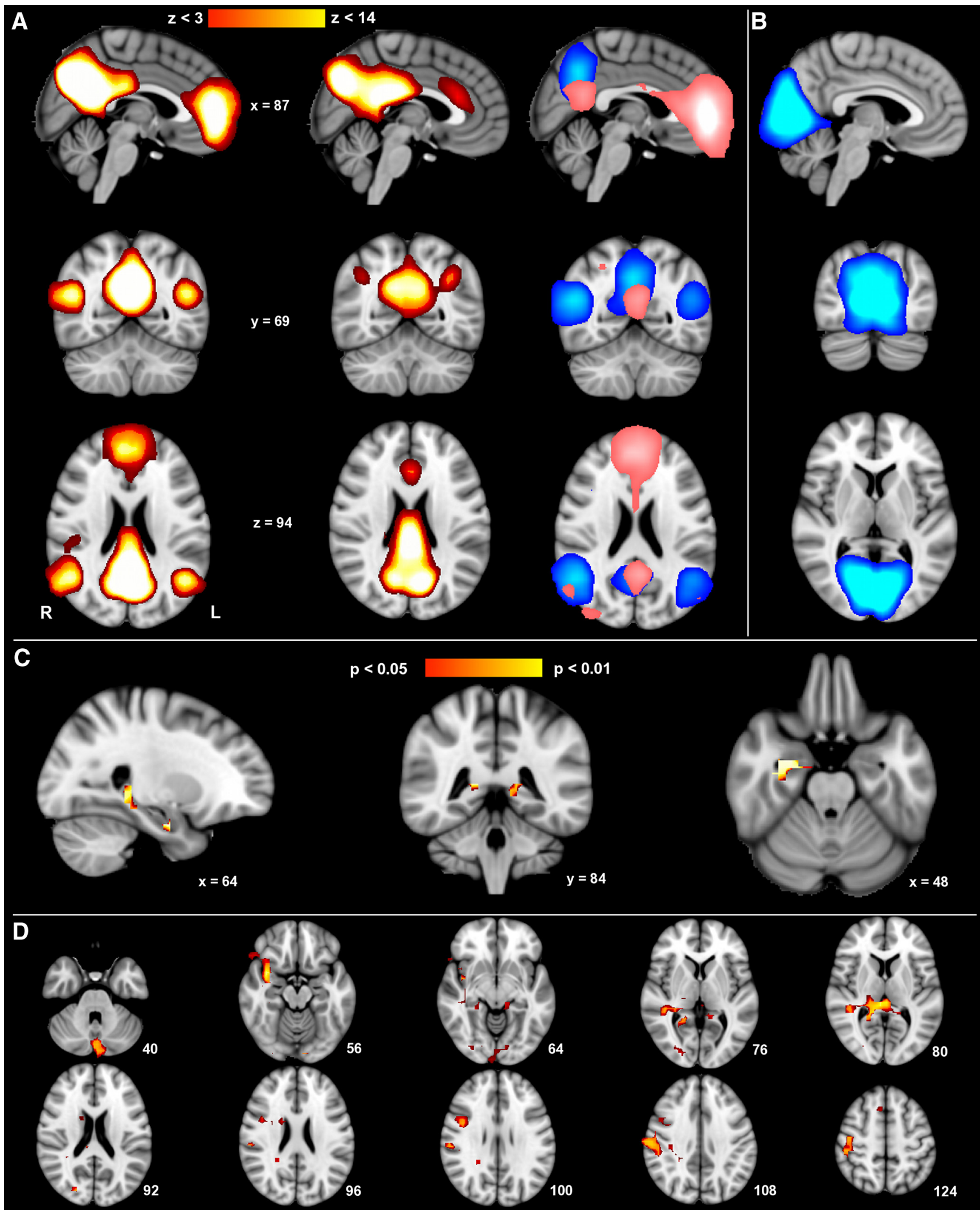

Figure 1. $A$, In red-yellow to the left is the DMN comprising three components from the gICA. In red-yellow to the right is the posterior DMN component in which we found increased functional synchronicity in APOE $\varepsilon 4$ carriers. This component spans ACC, PCC, precuneal, and thalamic regions. The two DMN components that showed no significant effect of APOE are shown in blue and pink. $\boldsymbol{B}$, The component spanning the primary visual cortex used as control. $\boldsymbol{C}$, Significant ( $p<0.05$, corrected) effects of APOE on the posterior DMN from the analysis restricted to hippocampal and amygdala areas. $\boldsymbol{D}$, Significant ( $p<0.05$, corrected) effects of APOE on the posterior DMN from the full-brain analysis. Numbers correspond to the $z$ value of each slice in $1 \mathrm{~mm}$ MNI-152 space. The effects extend into thalamic regions, posterior parts of hippocampus, lateral parts of the frontal lobe, the PCC, parietal cortex, and parahippocampal regions. All images are shown in radiological orientation, where the $x$ value increases in left direction, $y$ value increases in anterior direction, and $z$ value increases in superior direction. All images were transformed from 4 to $1 \mathrm{~mm}$ space for visualization purposes. 
Table 3. Increased synchronization with the posterior DMN

\begin{tabular}{|c|c|c|c|c|c|}
\hline Cluster & Volume $\left(\mathrm{mm}^{3}\right)$ & Mean (SD) APOE $\epsilon 4-$ & Mean (SD) APOE $\epsilon 4+$ & Cohen's d & $(x, y, z) \max$ \\
\hline \multicolumn{6}{|l|}{ HI/AM analysis $p \leq 0.05$} \\
\hline RHI/AM & 4608 & $0.03(2.86)$ & $4.83(4.70)$ & 1.23 & $(16,30,12)$ \\
\hline LHI/CWM & 832 & $1.49(5.67)$ & $6.83(6.43)$ & 0.88 & $(26,21,20)$ \\
\hline L AM/parahippocampal gyrus & 64 & $-1.48(8.56)$ & $5.69(9.05)$ & 0.81 & $(31,32,14)$ \\
\hline \multicolumn{6}{|l|}{ FB analysis $p \leq 0.05$} \\
\hline R insular/temporal pole & 19,328 & $0.42(3.09)$ & $6.27(5.90)$ & 1.24 & $(13,33,14)$ \\
\hline R supramarginal/postcentral & 9152 & $0.89(4.79)$ & $7.59(8.29)$ & 0.99 & $(8,26,27)$ \\
\hline L crus/vermis II & 5120 & $-2.32(7.09)$ & $8.64(12.6)$ & 1.07 & $(23,12,10)$ \\
\hline R lateral occipital/cuneal & 2816 & $0.97(3.26)$ & $5.39(5.24)$ & 1.01 & $(17,11,23)$ \\
\hline R precentral gyrus & 2304 & $-2.52(6.78)$ & $3.81(6.97)$ & 0.92 & $(12,32,25)$ \\
\hline R caudate/CWM & 512 & $-1.52(4.68)$ & $3.11(6.42)$ & 0.83 & $(18,32,24)$ \\
\hline R superior frontal/paracingulate gyrus & 192 & $-5.43(8.26)$ & $2.53(7.93)$ & 0.98 & $(21,36,31)$ \\
\hline RCWM & 128 & $-1.53(3.89)$ & $1.56(4.78)$ & 0.71 & $(15,32,24)$ \\
\hline Roccipital fusiform/lingual gyrus & 64 & $0.88(5.05)$ & $4.08(5.24)$ & 0.62 & $(18,11,16)$ \\
\hline
\end{tabular}

Significant clusters from the spatially restricted [hippocampus/amygdala (HI/AM)] and full-brain (FB) analysis, with respective cluster volumes, mean synchronization for carriers and noncarriers, Cohen's $d$ (effect of APOE genotype), and MNI coordinates of peak voxel within each cluster. The anatomical notation corresponds to the coordinate for the peak voxel according to the Harvard-0xford cortical and subcortical atlas. For the cluster with $(x, y, z)$ max $=(23,12,10)$ in the FB analysis, the cerebellar atlas in MNI-152 space after normalization with FNIRT was used. Cohen's $d$ was computed as (mean carrier - mean noncarrier)/SD pooled. CWM, Cortical white matter.

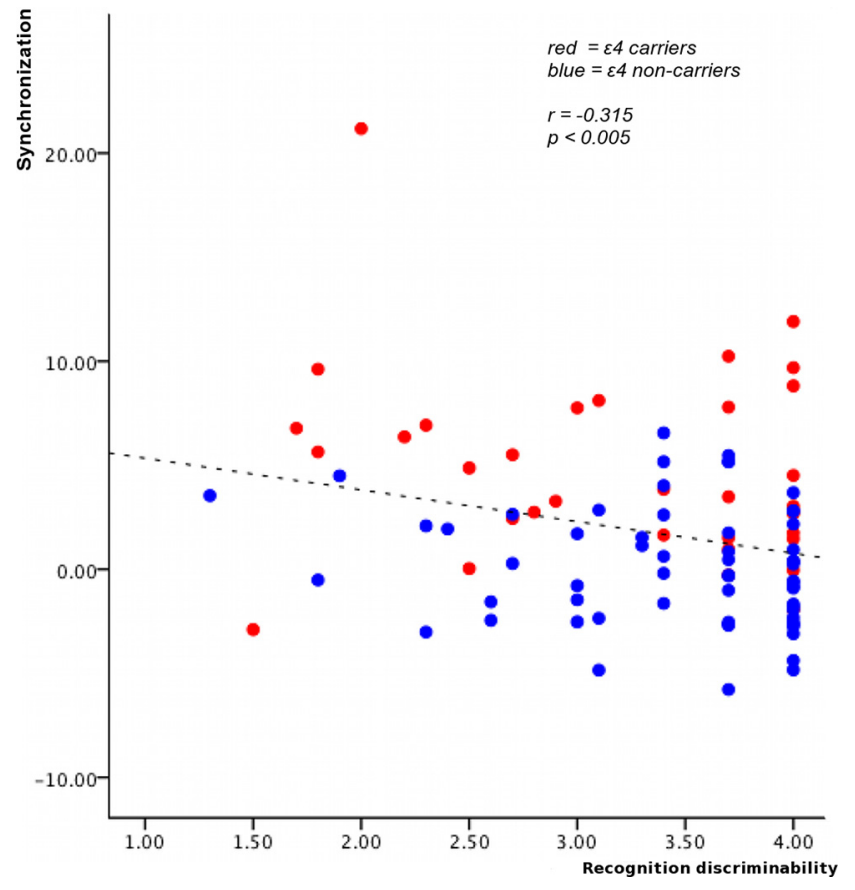

Figure 2. Total discriminability raw score plots as a function of hippocampal DMN synchronization. Red, Carriers; blue, noncarriers. The fit line illustrates the significant negative linear association $(r=-0.32, p<0.01$, partialing out age and sex). When we removed the outlier (synchronization $>20)$, the relationship was weaker but still significant $(r=-0.27, p<0.01)$.

$\beta(\mathrm{A} \beta)$ aggregation and clearance is more important than its fat-transport properties (Mahley and Rall, 2000; Kim et al., 2009; Herrup, 2010). It has been suggested that the main mechanism of $\mathrm{APOE}$ in $\mathrm{AD}$ is via the effects on $\mathrm{A} \beta$ metabolism (Kim et al., 2009). This is supported by findings of low concentrations of $\mathrm{A} \beta 42$ in the CSF of cognitively normal middle-aged $\varepsilon 4$ carriers (Sunderland et al., 2004), indicative of earlier cerebral amyloid deposition.

Previous studies have documented a connection between $\mathrm{A} \beta$ deposition and DMN connectivity. Pittsburgh Compound-B (PiB) (Klunk et al., 2004) PET studies have revealed retention of $\mathrm{A} \beta$ in posterior parietal regions near PCC and retrosplenial cortex in AD (Nordberg, 2004; Archer et al., 2006; Nordberg et al., 2010). Amyloid burden in posterior regions making up the memory network suggests a mechanism by which amyloid toxicity might disrupt memory function (Buckner et al., 2005). Similar $\mathrm{A} \beta$ deposition have been observed in subjects with increased genetic risk for AD (Reiman et al., 1996). A PET study by Buckner et al. (2005) demonstrated links between deposition of $A \beta$, cortical atrophy, and hypometabolism in PCC. This suggests that PCC is part of an intrinsic network specifically disrupted in early stages of $\mathrm{AD}$. This hypothesis is supported by three recent studies combining PiB-PET and fMRI (Hedden et al., 2009; Sperling et al., 2009; Sheline et al., 2010) reporting aberrant DMN activation in individuals with high amyloid burden. Consequently, increased DMN synchronization in $\varepsilon 4$ carriers may reflect increased $\mathrm{A} \beta$ deposition. However, without CSF biomarker data, such interpretations should be made with caution.

The functional significance of the observed increased hippocampal synchronicity with the DMN in carriers is not clear, but it seems reasonable to hypothesize that it could stem from a failure in hippocampal decoupling. A possible scenario is that aberrant hippocampal decoupling in $\varepsilon 4$ carriers results in increased hippocampal DMN synchronization during rest. This notion is partly supported by findings suggesting that age-related memory impairment may be primarily related to loss of deactivation in medial parietal regions (Miller et al., 2008). Also, a recent longitudinal study reported no difference in fMRI activity over 2 years in healthy individuals, whereas preclinical subjects demonstrated a decrease in hippocampal activity during the same period (O'Brien et al., 2010). Individuals showing more rapid cognitive decline demonstrated both increased hippocampal activation at baseline and accelerated longitudinal loss of hippocampal activation (O'Brien et al., 2010), suggesting that hippocampal hyperactivation is predictive of future cognitive decline. Decreased hippocampal decoupling during rest might increase the total hippocampal metabolic burden in carriers and modulate the present increased DMN synchronization. This is in line with our findings of a negative correlation between hippocampal DMN synchronization and performance on a test of memory function. We propose that aberrant hippocampal decoupling in $\varepsilon 4$ carriers might be linked to an increased $\mathrm{A} \beta$ burden. However, it remains unclear whether $\mathrm{A} \beta$ mainly exerts its effects via intracellular/intercellular signaling (Sanchez-Mejia et al., 2008), through decreased white matter myelination (Bartzokis et al., 2007), or by perturbing the receptor/ligand efficacy (Yun et al., 2005).

Whereas APOE $\varepsilon 4$ may modulate task-related BOLD signal independently of AD risk and pathology (Ringman et al., 2011), increased resting metabolic burden in the memory system of car- 
riers could be related to increased susceptibility of cognitive impairment later in life. This was partly supported by the negative correlation between the hippocampal DMN synchronization and memory performance in the current study and is consonant with a previous task-fMRI study (Celone et al., 2006). Because the DMN correlate negatively with task-related networks (Raichle and Snyder, 2007), increased hippocampal activity during rest might decrease hippocampal activation relative to baseline during memory tasks, explaining an apparent deficiency in memoryrelated hippocampal activation (Fleisher et al., 2009b). However, further studies delineating APOE effects on the functional interactions between task and rest are needed (Trachtenberg et al., 2010). Also, longitudinal studies are warranted to determine whether increased DMN synchronization is associated with higher risk of pathological brain and cognitive changes at a later stage.

The present study has limitations. Like most studies using convenience sampling, our sample represents a highly functioning subgroup of the population. The stability of the specific DMN component showing effect of APOE in the present analysis has been demonstrated in several studies (Damoiseaux et al., 2006; Buckner et al., 2008; Smith et al., 2009) and is almost identical to a component with high intrasession and intersession test-retest reliability (Zuo et al., 2010). At model order 42, this component showed the highest reproducibility along with a medial and lateral visual network (Zuo et al., 2010). This indicates a reliable intrinsic RSN.

However, choice of analytical approach could have influenced the results. Decreased DMN connectivity within MTL structures in elderly individuals with high amyloid burden has been demonstrated (Hedden et al., 2009; Sheline et al., 2010). These findings partly contrast with our and previous findings of increased DMN connectivity in $\varepsilon 4$ carriers (Filippini et al., 2009; Fleisher et al., 2009a). Importantly, the two former studies used seed-based correlation analysis, which differs from data-driven approaches in several aspects. ICA and dual regression represent a data-driven multivariate approach that effectively regresses out the common variance from all other included components, including RSNs showing various degree of spatial overlap. Furthermore, seeds based purely on anatomical definitions might yield results highly sensitive to the exact region of interest (ROI) placement and, therefore, vulnerable to operator-dependent variability and decreased interstudy reliability. This is in line with a recent comprehensive functional network analysis that demonstrated that functionally inaccurate ROIs may damage the network estimation, suggesting that results derived from inappropriate ROI definitions should be regarded with caution (Smith et al., 2011). Employing a data-driven approach like ICA in conjunction with dual regression might minimize the impact of inappropriate seeds.

In line with this reasoning, the present results are very similar to the findings of Filippini et al. (2009) using almost identical methods. This indicates reliable effects of genotype within the same analytical framework. However, we emphasize that the development of sensitive and reliable measures of functional connectivity is an active area of research, and future studies are needed to uncover the stability of APOE effects across analytical approaches and imaging modalities, including measures of microstructural integrity (Heise et al., 2010) and the relation to resting $\mathrm{BOLD}$ coherence. Several non-A $\beta$-mediated mechanisms are significant mediators of AD pathology (Herrup, 2010), and future studies might also be able to test the hypothesis that APOE effects are not specifically related to $\mathrm{AD}$ pathology but to some unidentified function of the gene, for example, on the cerebral vascular reactivity (Ringman et al., 2011).

Conclusively, we have documented increased resting state functional hippocampal coupling to the posterior DMN in healthy middle-aged and elderly carriers of the APOE $\varepsilon 4$ allele that could not be explained by hippocampal volumes or GM density. Posterior DMN synchronization was negatively correlated with memory performance, suggesting a neurocognitive significance of the findings. RSFC measures might thus be sensitive to genotypic differences related to increased risk of $A D$ several years before progression of $\mathrm{AD}$-related neurocognitive decline. Our findings are in agreement with the notion that APOE plays an important role in brain function, notably within the DMN and memory systems, which are tightly coupled to the loci of AD-related pathology. The hippocampal loci possibly reflect decreased decoupling during rest, which could in turn lead to elevated metabolic burden and increased vulnerability of the memory system in $\varepsilon 4$ carriers. The significant negative correlation between hippocampal DMN synchronization and memory performance supports this interpretation. Our findings provide an important replication and extension of specific genotype effects on intrinsic functional coherence, supporting the use of RSFC as an endophenotype in imaging genetics studies (Filippini et al., 2009; Glahn et al., 2010).

\section{References}

Andersson JLR, Jenkinson M, Smith S (2007a) Non-linear registration, aka spatial normalisation. FMRIB technical report TR07JA2.

Andersson JLR, Jenkinson M, Smith S (2007b) Non-linear optimisation. FMRIB technical report TR07JA1.

Archer HA, Edison P, Brooks DJ, Barnes J, Frost C, Yeatman T, Fox NC, Rossor MN (2006) Amyloid load and cerebral atrophy in Alzheimer's disease: an 11C-PIB positron emission tomography study. Ann Neurol 60:145-147.

Bartzokis G, Lu PH, Geschwind DH, Tingus K, Huang D, Mendez MF, Edwards N, Mintz J (2007) Apolipoprotein E affects both myelin breakdown and cognition: implications for age-related trajectories of decline into dementia. Biol Psychiatry 62:1380-1387.

Beckmann CF, Smith SM (2004) Probabilistic independent component analysis for functional magnetic resonance imaging. IEEE Trans Med Imaging 23:137-152.

Beckmann CF, DeLuca M, Devlin JT, Smith SM (2005) Investigations into resting-state connectivity using independent component analysis. Philos Trans R Soc Lond B Biol Sci 360:1001-1013.

Benjamin R, Leake A, McArthur FK, Ince PG, Candy JM, Edwardson JA, Morris CM, Bjertness E (1994) Protective effect of apoE epsilon 2 in Alzheimer's disease. Lancet 344:473.

Bertram L, McQueen MB, Mullin K, Blacker D, Tanzi RE (2007) Systematic meta-analyses of Alzheimer disease genetic association studies: the AlzGene database. Nat Genet 39:17-23.

Biswal B, Yetkin FZ, Haughton VM, Hyde JS (1995) Functional connectivity in the motor cortex of resting human brain using echo-planar MRI. Magn Reson Med 34:537-541.

Boly M, Phillips C, Tshibanda L, Vanhaudenhuyse A, Schabus M, Dang-Vu TT, Moonen G, Hustinx R, Maquet P, Laureys S (2008) Intrinsic brain activity in altered states of consciousness: how conscious is the default mode of brain function? Ann N Y Acad Sci 1129:119-129.

Braak H, Braak E (1991) Neuropathological stageing of Alzheimer-related changes. Acta Neuropathol 82:239-259.

Buckner RL, Head D, Parker J, Fotenos AF, Marcus D, Morris JC, Snyder AZ (2004) A unified approach for morphometric and functional data analysis in young, old, and demented adults using automated atlas-based head size normalization: reliability and validation against manual measurement of total intracranial volume. Neuroimage 23:724-738.

Buckner RL, Snyder AZ, Shannon BJ, LaRossa G, Sachs R, Fotenos AF, Sheline YI, Klunk WE, Mathis CA, Morris JC, Mintun MA (2005) Molecular, structural, and functional characterization of Alzheimer's disease: evidence for a relationship between default activity, amyloid, and memory. J Neurosci 25:7709-7717. 
Buckner RL, Andrews-Hanna JR, Schacter DL (2008) The brain's default network: anatomy, function, and relevance to disease. Ann N Y Acad Sci 1124:1-38.

Celone KA, Calhoun VD, Dickerson BC, Atri A, Chua EF, Miller SL, DePeau K, Rentz DM, Selkoe DJ, Blacker D, Albert MS, Sperling RA (2006) Alterations in memory networks in mild cognitive impairment and Alzheimer's disease: an independent component analysis. J Neurosci 26:10222-10231.

Cohen J (1992) A power primer. Psychol Bull 112:155-159.

Dale AM, Sereno MI (1993) Improved localization of cortical activity by combining EEG and MEG with MRI cortical surface reconstruction: a linear approach. J Cogn Neurosci 5:162-176.

Dale AM, Fischl B, Sereno MI (1999) Cortical surface-based analysis. I. Segmentation and surface reconstruction. Neuroimage 9:179-194.

Damoiseaux JS, Rombouts SA, Barkhof F, Scheltens P, Stam CJ, Smith SM, Beckmann CF (2006) Consistent resting-state networks across healthy subjects. Proc Natl Acad Sci U S A 103:13848-13853.

Damoiseaux JS, Beckmann CF, Arigita EJ, Barkhof F, Scheltens P, Stam CJ, Smith SM, Rombouts SA (2008) Reduced resting-state brain activity in the "default network" in normal aging. Cereb Cortex 18:1856-1864.

Deary IJ, Whiteman MC, Pattie A, Starr JM, Hayward C, Wright AF, Carothers A, Whalley LJ (2002) Cognitive change and the APOE epsilon 4 allele. Nature 418:932.

Delis DC, Kramer JH, Kaplan E, Ober BA (1987) California Verbal Learning Test. San Antonio, TX: Psychological Corporation.

Espeseth T, Westlye LT, Fjell AM, Walhovd KB, Rootwelt H, Reinvang I (2008) Accelerated age-related cortical thinning in healthy carriers of apolipoprotein E epsilon 4. Neurobiol Aging 29:329-340.

Filippini N, MacIntosh BJ, Hough MG, Goodwin GM, Frisoni GB, Smith SM, Matthews PM, Beckmann CF, Mackay CE (2009) Distinct patterns of brain activity in young carriers of the APOE-epsilon4 allele. Proc Natl Acad Sci U S A 106:7209-7214.

Fischl B, Dale AM (2000) Measuring the thickness of the human cerebral cortex from magnetic resonance images. Proc Natl Acad Sci U S A 97:11050-11055.

Fischl B, Liu A, Dale AM (2001) Automated manifold surgery: constructing geometrically accurate and topologically correct models of the human cerebral cortex. IEEE Trans Med Imaging 20:70-80.

Fischl B, Salat DH, Busa E, Albert M, Dieterich M, Haselgrove C, van der Kouwe A, Killiany R, Kennedy D, Klaveness S, Montillo A, Makris N, Rosen B, Dale AM (2002) Whole brain segmentation: automated labeling of neuroanatomical structures in the human brain. Neuron 33:341-355.

Fischl B, Salat DH, van der Kouwe AJ, Makris N, Segonne F, Quinn BT, Dale AM (2004) Sequence-independent segmentation of magnetic resonance images. Neuroimage 23 [Suppl 1]:S69-S84.

Fleisher AS, Sherzai A, Taylor C, Langbaum JB, Chen K, Buxton RB (2009a) Resting-state BOLD networks versus task-associated functional MRI for distinguishing Alzheimer's disease risk groups. Neuroimage 47: $1678-1690$.

Fleisher AS, Podraza KM, Bangen KJ, Taylor C, Sherzai A, Sidhar K, Liu TT, Dale AM, Buxton RB (2009b) Cerebral perfusion and oxygenation differences in Alzheimer's disease risk. Neurobiol Aging 30:1737-1748.

Folstein MF, Folstein SE, McHugh PR (1975) “Mini-mental state.” A practical method for grading the cognitive state of patients for the clinician. J Psychiatr Res 12:189-198.

Fox MD, Snyder AZ, Vincent JL, Corbetta M, Van Essen DC, Raichle ME (2005) The human brain is intrinsically organized into dynamic, anticorrelated functional networks. Proc Natl Acad Sci U S A 102:9673-9678.

Fransson P, Skiöld B, Horsch S, Nordell A, Blennow M, Lagercrantz H, Aden U (2007) Resting-state networks in the infant brain. Proc Natl Acad Sci U S A 104:15531-15536.

Fransson P, Aden U, Blennow M, Lagercrantz H (2011) The functional architecture of the infant brain as revealed by resting-state fMRI. Cereb Cortex 21:145-154.

Gerdes LU (2003) The common polymorphism of apolipoprotein E: geographical aspects and new pathophysiological relations. Clin Chem Lab Med 41:628-631.

Glahn DC, Winkler AM, Kochunov P, Almasy L, Duggirala R, Carless MA, Curran JC, Olvera RL, Laird AR, Smith SM, Beckmann CF, Fox PT, Blangero J (2010) Genetic control over the resting brain. Proc Natl Acad Sci U S A 107:1223-1228.

Greicius MD, Krasnow B, Reiss AL, Menon V (2003) Functional connectiv- ity in the resting brain: a network analysis of the default mode hypothesis. Proc Natl Acad Sci U S A 100:253-258.

Greicius MD, Srivastava G, Reiss AL, Menon V (2004) Default-mode network activity distinguishes Alzheimer's disease from healthy aging: evidence from functional MRI. Proc Natl Acad Sci U S A 101:4637-4642.

Hedden T, Van Dijk KR, Becker JA, Mehta A, Sperling RA, Johnson KA, Buckner RL (2009) Disruption of functional connectivity in clinically normal older adults harboring amyloid burden. J Neurosci 29:12686-12694.

Heise V, Filippini N, Ebmeier KP, Mackay CE (2010) The APOE varepsilon4 allele modulates brain white matter integrity in healthy adults. Mol Psychiatry. Advance online publication. Retrieved Nov. 10, 2010. doi:10.1038/mp. 2010.90 .

Herrup K (2010) Reimagining Alzheimer's disease—an age-based hypothesis. J Neurosci 30:16755-16762.

Jenkinson M, Smith S (2001) A global optimisation method for robust affine registration of brain images. Med Image Anal 5:143-156.

Jenkinson M, Bannister P, Brady M, Smith S (2002) Improved optimization for the robust and accurate linear registration and motion correction of brain images. Neuroimage 17:825-841.

Kim J, Basak JM, Holtzman DM (2009) The role of apolipoprotein E in Alzheimer's disease. Neuron 63:287-303.

Klunk WE, Engler H, Nordberg A, Wang Y, Blomqvist G, Holt DP, Bergström M, Savitcheva I, Huang GF, Estrada S, Ausén B, Debnath ML, Barletta J, Price JC, Sandell J, Lopresti BJ, Wall A, Koivisto P, Antoni G, Mathis CA, et al. (2004) Imaging brain amyloid in Alzheimer's disease with Pittsburgh Compound-B. Ann Neurol 55:306-319.

Mahley RW, Rall SC Jr (2000) Apolipoprotein E: far more than a lipid transport protein. Annu Rev Genomics Hum Genet 1:507-537.

Margulies DS, Vincent JL, Kelly C, Lohmann G, Uddin LQ, Biswal BB, Villringer A, Castellanos FX, Milham MP, Petrides M (2009) Precuneus shares intrinsic functional architecture in humans and monkeys. Proc Natl Acad Sci U S A 106:20069-20074.

Miller SL, Celone K, DePeau K, Diamond E, Dickerson BC, Rentz D, Pihlajamäki M, Sperling RA (2008) Age-related memory impairment associated with loss of parietal deactivation but preserved hippocampal activation. Proc Natl Acad Sci U S A 105:2181-2186.

Minka TP (2000) Automatic choice of dimensionality for PCA. Cambridge, MA: MIT Media Laboratory, Vision and Modeling Group.

Nichols TE, Holmes AP (2002) Nonparametric permutation tests for functional neuroimaging: a primer with examples. Hum Brain Mapp 15:1-25.

Nordberg A (2004) PET imaging of amyloid in Alzheimer's disease. Lancet Neurol 3:519-527.

Nordberg A, Rinne JO, Kadir A, Långström B (2010) The use of PET in Alzheimer disease. Nat Rev Neurol 6:78-87.

O'Brien JL, O'Keefe KM, LaViolette PS, DeLuca AN, Blacker D, Dickerson BC, Sperling RA (2010) Longitudinal fMRI in elderly reveals loss of hippocampal activation with clinical decline. Neurology 74:1969-1976.

Persson J, Lind J, Larsson A, Ingvar M, Sleegers K, Van Broeckhoven C, Adolfsson R, Nilsson LG, Nyberg L (2008) Altered deactivation in individuals with genetic risk for Alzheimer's disease. Neuropsychologia 46:1679-1687.

Pihlajamäki M, Sperling RA (2009) Functional MRI assessment of taskinduced deactivation of the default mode network in Alzheimer's disease and at-risk older individuals. Behav Neurol 21:77-91.

Raichle ME, Snyder AZ (2007) A default mode of brain function: a brief history of an evolving idea. Neuroimage 37:1083-1090; discussion 1097-1099.

Raichle ME, MacLeod AM, Snyder AZ, Powers WJ, Gusnard DA, Shulman GL (2001) A default mode of brain function. Proc Natl Acad Sci U S A 98:676-682.

Reiman EM, Caselli RJ, Yun LS, Chen K, Bandy D, Minoshima S, Thibodeau SN, Osborne D (1996) Preclinical evidence of Alzheimer's disease in persons homozygous for the epsilon 4 allele for apolipoprotein E. N Engl J Med 334:752-758.

Ringman JM, Medina LD, Braskie M, Rodriguez-Agudelo Y, Geschwind DH Macias-Islas MA, Cummings JL, Bookheimer S (2011) Effects of risk genes on BOLD activation in presymptomatic carriers of familial Alzheimer's disease mutations during a novelty encoding task. Cereb Cortex 21:877-883.

Roosendaal SD, Schoonheim MM, Hulst HE, Sanz-Arigita EJ, Smith SM, Geurts JJ, Barkhof F (2010) Resting state networks change in clinically isolated syndrome. Brain 133:1612-1621. 
Roses AD (1996) Apolipoprotein E alleles as risk factors in Alzheimer's disease. Annu Rev Med 47:387-400.

Sanchez-Mejia RO, Newman JW, Toh S, Yu GQ, Zhou Y, Halabisky B, Cissé M, Scearce-Levie K, Cheng IH, Gan L, Palop JJ, Bonventre JV, Mucke L (2008) Phospholipase A2 reduction ameliorates cognitive deficits in a mouse model of Alzheimer's disease. Nat Neurosci 11:1311-1318.

Ségonne F, Dale AM, Busa E, Glessner M, Salat D, Hahn HK, Fischl B (2004) A hybrid approach to the skull stripping problem in MRI. Neuroimage 22:1060-1075.

Ségonne F, Pacheco J, Fischl B (2007) Geometrically accurate topologycorrection of cortical surfaces using nonseparating loops. IEEE Trans Med Imaging 26:518-529.

Sheline YI, Raichle ME, Snyder AZ, Morris JC, Head D, Wang S, Mintun MA (2010) Amyloid plaques disrupt resting state default mode network connectivity in cognitively normal elderly. Biol Psychiatry 67:584-587.

Sled JG, Zijdenbos AP, Evans AC (1998) A nonparametric method for automatic correction of intensity nonuniformity in MRI data. IEEE Trans Med Imaging 17:87-97.

Small GW, Ercoli LM, Silverman DH, Huang SC, Komo S, Bookheimer SY, Lavretsky H, Miller K, Siddarth P, Rasgon NL, Mazziotta JC, Saxena S, Wu HM, Mega MS, Cummings JL, Saunders AM, Pericak-Vance MA, Roses AD, Barrio JR, Phelps ME (2000) Cerebral metabolic and cognitive decline in persons at genetic risk for Alzheimer's disease. Proc Natl Acad Sci U S A 97:6037-6042.

Smith SM, Nichols TE (2009) Threshold-free cluster enhancement: addressing problems of smoothing, threshold dependence and localisation in cluster inference. Neuroimage 44:83-98.

Smith SM, Jenkinson M, Woolrich MW, Beckmann CF, Behrens TE, Johansen-Berg H, Bannister PR, De Luca M, Drobnjak I, Flitney DE, Niazy RK, Saunders J, Vickers J, Zhang Y, De Stefano N, Brady JM, Matthews PM (2004) Advances in functional and structural MR image analysis and implementation as FSL. Neuroimage 23 [Suppl 1]:S208-S219.

Smith SM, Fox PT, Miller KL, Glahn DC, Fox PM, Mackay CE, Filippini N, Watkins KE, Toro R, Laird AR, Beckmann CF (2009) Correspondence of the brain's functional architecture during activation and rest. Proc Natl Acad Sci U S A 106:13040-13045.

Smith SM, Miller KL, Salimi-Khorshidi G, Webster M, Beckmann CF, Nichols TE, Ramsey JD, Woolrich MW (2011) Network modelling methods for fMRI. Neuroimage 54:875-891.
Sorg C, Riedl V, Mühlau M, Calhoun VD, Eichele T, Läer L, Drzezga A, Förstl H, Kurz A, Zimmer C, Wohlschläger AM (2007) Selective changes of resting-state networks in individuals at risk for Alzheimer's disease. Proc Natl Acad Sci U S A 104:18760-18765.

Sperling RA, Laviolette PS, O'Keefe K, O’Brien J, Rentz DM, Pihlajamaki M, Marshall G, Hyman BT, Selkoe DJ, Hedden T, Buckner RL, Becker JA, Johnson KA (2009) Amyloid deposition is associated with impaired default network function in older persons without dementia. Neuron 63:178-188.

Sunderland T, Mirza N, Putnam KT, Linker G, Bhupali D, Durham R, Soares H, Kimmel L, Friedman D, Bergeson J, Csako G, Levy JA, Bartko JJ, Cohen RM (2004) Cerebrospinal fluid beta-amyloidl-42 and tau in control subjects at risk for Alzheimer's disease: the effect of APOE epsilon4 allele. Biol Psychiatry 56:670-676.

Trachtenberg AJ, Filippini N, Mackay CE (2010) The effects of APOEepsilon 4 on the BOLD response. Neurobiol Aging. Advance online publication. Retrieved May 18, 2010. doi:10.1016/j.neurobiolaging.2010.03.009.

Vincent JL, Patel GH, Fox MD, Snyder AZ, Baker JT, Van Essen DC, Zempel JM, Snyder LH, Corbetta M, Raichle ME (2007) Intrinsic functional architecture in the anaesthetized monkey brain. Nature 447:83-86.

Wilson RS, Bienias JL, Berry-Kravis E, Evans DA, Bennett DA (2002) The apolipoprotein E epsilon 2 allele and decline in episodic memory. J Neurol Neurosurg Psychiatry 73:672-677.

Woolrich MW, Jbabdi S, Patenaude B, Chappell M, Makni S, Behrens T, Beckmann C, Jenkinson M, Smith SM (2009) Bayesian analysis of neuroimaging data in FSL. Neuroimage 45:S173-186.

Yun SH, Park KA, Sullivan P, Pasternak JF, Ladu MJ, Trommer BL (2005) Blockade of nicotinic acetylcholine receptors suppresses hippocampal long-term potentiation in wild-type but not ApoE4 targeted replacement mice. J Neurosci Res 82:771-777.

Zhang D, Raichle ME (2010) Disease and the brain's dark energy. Nat Rev Neurol 6:15-28.

Zhang Y, Brady M, Smith S (2001) Segmentation of brain MR images through a hidden Markov random field model and the expectationmaximization algorithm. IEEE Trans Med Imaging 20:45-57.

Zuo XN, Kelly C, Adelstein JS, Klein DF, Castellanos FX, Milham MP (2010) Reliable intrinsic connectivity networks: test-retest evaluation using ICA and dual regression approach. Neuroimage 49:2163-2177. 